\section{THE X-RAY STRUCTURE DETERMINATION OF OXANOSINE}

Sir:

In the previous paper ${ }^{1)}$, the isolation and chemical and biological properties of oxanosine were reported. In this paper the X-ray structure determination of oxanosine is described.

Colorless, thin, platy crystals of oxanosine were grown in ethanol-water mixed solutions. A sample of approximate dimensions $0.4 \times 0.08 \times$ $0.02 \mathrm{~mm}$ was mounted on a Philips PW1100 diffractometer. The lattice constants and intensity data were obtained using $\mathrm{CuK} \alpha$ radiation monochromated by a graphite plate. 2753 reflections were measured by the $\theta-2 \theta$ scan method with a scan speed of $6^{\circ}(2 \theta) / \mathrm{sec}$ within the $2 \theta$ range $0^{\circ}$ to $156^{\circ}$; of these measurements, 2043 were above the $2 \sigma(\mathrm{I})$ level and were used for the structure determination. The crystal data are given in Table 1. The asymmetric unit contains two independent oxanosine molecules A and B and one water molecule.

The structure was solved by direct methods using the MULTAN program ${ }^{2)}$ and refined by

Table 1. Crystal data of oxanosine hemihydrate.

Oxanosine hemihydrate, $\mathrm{C}_{10} \mathrm{H}_{12} \mathrm{~N}_{4} \mathrm{O}_{6} \cdot \frac{1}{2} \mathrm{H}_{2} \mathrm{O}$,

F.W. $=293.2$

Monoclinic $\mathrm{P} 2_{1}, \mathrm{Z}=4, \mathrm{D}_{\mathrm{ca} 1}=1.634 \mathrm{gcm}^{-3}$

$a=25.562$ (12), $b=8.973$ (5), $c=5.219$ (3) $\AA$, $\beta=94.88(5)^{\circ}, U=1192.7 \AA^{3}$. least-squares procedures. All the hydrogen atoms except those of water were located on a differenceelectrondensity map and their coordinates and isotropic temperature factors were subjected to the refinement. The final $\mathrm{R}$ value was $4.8 \%$ *

The absolute configuration was determined chemically by isolation of D-ribose $\left([\alpha]_{D}^{22}-19^{\circ}\right.$, c $0.5, \mathrm{H}_{2} \mathrm{O}$ ) from the acid hydrolysate of oxanosine with $0.1 \mathrm{~N} \mathrm{H}_{2} \mathrm{SO}_{4}$ at $100^{\circ} \mathrm{C}$ for 4 hours. The stereochemistry was confirmed further by transformation of oxanosine into xanthosine under mild alkaline conditions (1.2 eq. $\mathrm{KOH}$ in $50 \%$ acetone at $8^{\circ} \mathrm{C}$ for 10 days, $15.3 \%$ yield after purification).

The bond lengths and angles of the molecules $\mathrm{A}$ and $\mathbf{B}$ are compared in Tables 2 and 3. The values are consistent with the chemical structure shown in Fig. 1. The location of the hydrogen atoms also supports this structure. From Tables

Fig. 1. Structure of oxanosine.

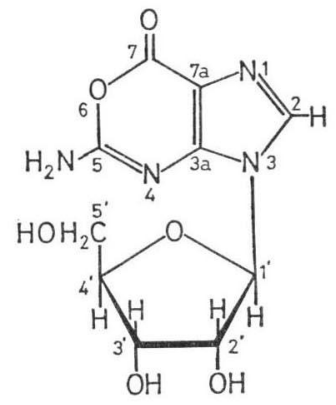

5-Amino-3- $\beta$-D-ribofuranosyl-3 $H$-imidazo[4,5d] $[1,3]$ oxazin-7-one

Table 2. Comparison of bond lengths between molecules A and B.

\begin{tabular}{l|cc||c|cc}
\hline & Molecule A & Molecule B & & Molecule A & Molecule B \\
\hline $\mathrm{C}(5)-\mathrm{O}(6)$ & $1.363(6)$ & $1.358(6)$ & $\mathrm{C}(2)-\mathrm{N}(3)$ & $1.378(7)$ & $1.379(6)$ \\
$\mathrm{C}(7)-\mathrm{O}(6)$ & $1.404(6)$ & $1.428(7)$ & $\mathrm{C}\left(1^{\prime}\right)-\mathrm{N}(3)$ & $1.483(6)$ & $1.462(6)$ \\
$\mathrm{C}(5)-\mathrm{N}(4)$ & $1.310(7)$ & $1.321(7)$ & $\mathrm{C}\left(1^{\prime}\right)-\mathrm{C}\left(2^{\prime}\right)$ & $1.520(7)$ & $1.558(7)$ \\
$\mathrm{C}(5)-\mathrm{N}(5)$ & $1.335(8)$ & $1.322(7)$ & $\mathrm{C}\left(1^{\prime}\right)-\mathrm{O}\left(1^{\prime}\right)$ & $1.410(6)$ & $1.407(6)$ \\
$\mathrm{C}(3 \mathrm{a})-\mathrm{N}(4)$ & $1.358(7)$ & $1.360(6)$ & $\mathrm{C}\left(2^{\prime}\right)-\mathrm{C}\left(3^{\prime}\right)$ & $1.538(8)$ & $1.534(7)$ \\
$\mathrm{C}(3 \mathrm{a})-\mathrm{C}(7 \mathrm{a})$ & $1.386(7)$ & $1.386(6)$ & $\mathrm{C}\left(2^{\prime}\right)-\mathrm{O}\left(2^{\prime}\right)$ & $1.420(6)$ & $1.409(7)$ \\
$\mathrm{C}(3 \mathrm{a})-\mathrm{N}(3)$ & $1.372(7)$ & $1.364(7)$ & $\mathrm{C}\left(3^{\prime}\right)-\mathrm{C}\left(4^{\prime}\right)$ & $1.534(7)$ & $1.510(7)$ \\
$\mathrm{C}(7 \mathrm{a})-\mathrm{C}(7)$ & $1.414(7)$ & $1.419(8)$ & $\mathrm{C}\left(3^{\prime}\right)-\mathrm{O}\left(3^{\prime}\right)$ & $1.405(6)$ & $1.436(6)$ \\
$\mathrm{C}(7 \mathrm{a})-\mathrm{N}(1)$ & $1.398(7)$ & $1.379(7)$ & $\mathrm{C}\left(4^{\prime}\right)-\mathrm{O}\left(1^{\prime}\right)$ & $1.460(6)$ & $1.446(7)$ \\
$\mathrm{C}(7)-\mathrm{O}(7)$ & $1.207(6)$ & $1.190(6)$ & $\mathrm{C}\left(4^{\prime}\right)-\mathrm{C}\left(5^{\prime}\right)$ & $1.515(8)$ & $1.520(8)$ \\
$\mathrm{C}(2)-\mathrm{N}(1)$ & $1.310(6)$ & $1.309(7)$ & $\mathrm{C}\left(5^{\prime}\right)-\mathrm{O}\left(5^{\prime}\right)$ & $1.424(8)$ & $1.434(7)$ \\
\hline
\end{tabular}

\footnotetext{
* The list of the atomic parameters will be obtained from Y. IITAKA upon request.
} 
Table 3. Comparison of bond angles between molecules A and B.

\begin{tabular}{|c|c|c|c|c|c|}
\hline & Molecule A & Molecule B & & Molecule A & Molecule B \\
\hline$C(5)-O(6)-C(7)$ & $124.1(4)$ & $124.6(4)$ & $C(2)-N(3)-C\left(1^{\prime}\right)$ & $126.4(4)$ & $128.2(4)$ \\
\hline $\mathrm{N}(4)-\mathrm{C}(5)-\mathrm{O}(6)$ & $125.1(5)$ & $125.4(5)$ & $\mathrm{C}(3 \mathrm{a})-\mathrm{N}(3)-\mathrm{C}(2)$ & $106.8(4)$ & $106.2(4)$ \\
\hline $\mathrm{N}(4)-\mathrm{C}(5)-\mathrm{N}(5)$ & $122.5(5)$ & $122.7(5)$ & $\mathrm{N}(3)-\mathrm{C}\left(1^{\prime}\right)-\mathrm{C}\left(2^{\prime}\right)$ & $113.1(4)$ & 112.9 (4) \\
\hline $\mathrm{O}(6)-\mathrm{C}(5)-\mathrm{N}(5)$ & $112.3(4)$ & $111.9(4)$ & $\mathrm{C}\left(2^{\prime}\right)-\mathrm{C}\left(1^{\prime}\right)-\mathrm{O}\left(1^{\prime}\right)$ & $106.5(4)$ & $107.5(4)$ \\
\hline $\mathrm{C}(3 \mathrm{a})-\mathrm{N}(4)-\mathrm{C}(5)$ & $112.1(4)$ & $111.6(4)$ & $\mathrm{N}(3)-\mathrm{C}\left(1^{\prime}\right)-\mathrm{O}\left(1^{\prime}\right)$ & $107.6(4)$ & $110.1(4)$ \\
\hline$C(7 a)-C(3 a)-N(4)$ & $127.7(5)$ & $127.8(5)$ & $C\left(3^{\prime}\right)-C\left(2^{\prime}\right)-C\left(1^{\prime}\right)$ & $101.4(4)$ & $101.7(4)$ \\
\hline $\mathrm{C}(7 \mathrm{a})-\mathrm{C}(3 \mathrm{a})-\mathrm{N}(3)$ & $105.4(4)$ & $106.0(4)$ & $\mathrm{C}\left(3^{\prime}\right)-\mathrm{C}\left(2^{\prime}\right)-\mathrm{O}\left(2^{\prime}\right)$ & $108.7(4)$ & $113.1(4)$ \\
\hline$N(4)-C(3 a)-N(3)$ & $127.0(5)$ & $126.2(4)$ & $\mathrm{C}\left(1^{\prime}\right)-\mathrm{C}\left(2^{\prime}\right)-\mathrm{O}\left(2^{\prime}\right)$ & $107.8(4)$ & $111.9(4)$ \\
\hline$C(7)-C(7 a)-C(3 a)$ & $119.0(5)$ & $120.2(5)$ & $\mathrm{C}\left(4^{\prime}\right)-\mathrm{C}\left(3^{\prime}\right)-\mathrm{C}\left(2^{\prime}\right)$ & $102.2(4)$ & $103.3(4)$ \\
\hline $\mathrm{C}(7)-\mathrm{C}(7 \mathrm{a})-\mathrm{N}(1)$ & $130.4(5)$ & $129.6(5)$ & $\mathrm{C}\left(4^{\prime}\right)-\mathrm{C}\left(3^{\prime}\right)-\mathrm{O}\left(3^{\prime}\right)$ & $113.9(4)$ & $109.1(4)$ \\
\hline $\mathrm{C}(3 \mathrm{a})-\mathrm{C}(7 \mathrm{a})-\mathrm{N}(1)$ & $110.6(5)$ & $110.2(4)$ & $\mathrm{C}\left(2^{\prime}\right)-\mathrm{C}\left(3^{\prime}\right)-\mathrm{O}\left(3^{\prime}\right)$ & $113.5(4)$ & $110.7(4)$ \\
\hline $\mathrm{O}(7)-\mathrm{C}(7)-\mathrm{O}(6)$ & $115.7(5)$ & $116.8(5)$ & $\mathrm{O}\left(1^{\prime}\right)-\mathrm{C}\left(4^{\prime}\right)-\mathrm{C}\left(3^{\prime}\right)$ & $105.5(4)$ & $105.9(4)$ \\
\hline $\mathrm{O}(7)-\mathrm{C}(7)-\mathrm{C}(7 \mathrm{a})$ & $132.5(5)$ & $132.9(5)$ & $\mathrm{O}\left(1^{\prime}\right)-\mathrm{C}\left(4^{\prime}\right)-\mathrm{C}\left(5^{\prime}\right)$ & $106.7(4)$ & $107.9(4)$ \\
\hline$O(6)-C(7)-C(7 a)$ & $111.8(4)$ & $110.3(4)$ & $\mathrm{C}\left(3^{\prime}\right)-\mathrm{C}\left(4^{\prime}\right)-\mathrm{C}\left(5^{\prime}\right)$ & $114.6(4)$ & $114.7(4)$ \\
\hline $\mathrm{C}(2)-\mathrm{N}(1)-\mathrm{C}(7 \mathrm{a})$ & $104.2(4)$ & $104.8(4)$ & $\mathrm{O}\left(5^{\prime}\right)-\mathrm{C}\left(5^{\prime}\right)-\mathrm{C}\left(4^{\prime}\right)$ & $111.2(5)$ & $112.1(4)$ \\
\hline $\mathrm{N}(3)-\mathrm{C}(2)-\mathrm{N}(1)$ & $113.0(5)$ & $112.7(5)$ & $\mathrm{C}\left(1^{\prime}\right)-\mathrm{O}\left(1^{\prime}\right)-\mathrm{C}\left(4^{\prime}\right)$ & $110.2(4)$ & $110.6(4)$ \\
\hline $\mathrm{C}(3 \mathrm{a})-\mathrm{N}(3)-\mathrm{C}\left(1^{\prime}\right)$ & $126.4(4)$ & $125.5(4)$ & & & \\
\hline
\end{tabular}

2 and 3 , it is clear that significantly different (more than $3 \sigma$ level) values are found for parameters involving the atoms $\mathrm{N}(3), \mathrm{C}\left(1^{\prime}\right), \mathrm{C}\left(2^{\prime}\right)$ and $\mathrm{C}\left(3^{\prime}\right)$. This may be caused by the difference in the angles of twist about the glycosyl bonds and in the direction of the hydrogen bonds involving the atoms $\mathrm{O}\left(2^{\prime}\right)$ and $\mathrm{O}\left(3^{\prime}\right)$.

Fig. 2 shows the conformation of the molecules $A$ and $B$ in an ORTEP drawing ${ }^{3)}$. Various conformation angles are compared in Table 4. The angle $\chi$ about the glycosyl bond lies in the range described as anti in both molecules although the values are greatly differ $\left(\mathrm{A},-12.3^{\circ} ; \mathrm{B}, 79.3^{\circ}\right)$.

Fig. 2. A perspective drawing of the molecules A and $\mathrm{B}$ of oxanosine.
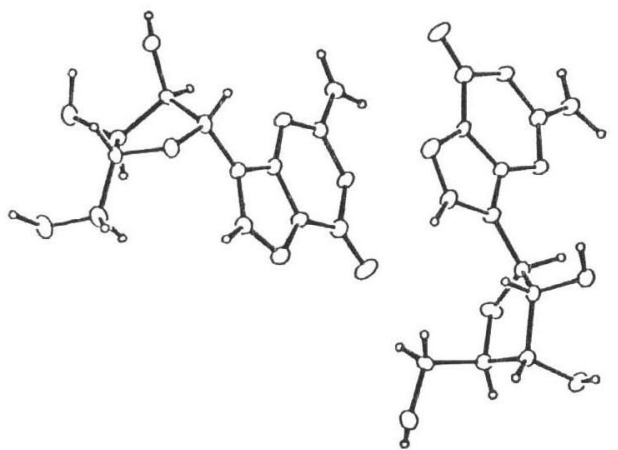

A

B
The difference is probably caused by the difference in inter-molecular hydrogen bonds. Both $\mathrm{C}\left(2^{\prime}\right)$ exo and $\mathrm{C}\left(3^{\prime}\right)$-exo conformations of the furanose ring found for molecules A and B, respectively, are not common in ribonucleosides and ribonucleotides $^{4)}$. The $\mathrm{C}\left(4^{\prime}\right)-\mathrm{C}\left(5^{\prime}\right)$ bond takes the $\mathrm{tg}^{-}$ conformation in both molecules which is also rather rare even in nucleosides. The unusual conformations all undoubtedly originate from the particular intermolecular hydrogen bonds includ-

Table 4. Torsional angles in furanose ring.

\begin{tabular}{|c|c|c|}
\hline Angle & \multicolumn{2}{|c|}{ Molecule A Molecule B } \\
\hline$\chi\left[\mathrm{C}(2)-\mathrm{N}(3)-\mathrm{C}\left(1^{\prime}\right)-\mathrm{O}\left(1^{\prime}\right)\right]$ & $-12.3^{\circ}$ & $79.3^{\circ}$ \\
\hline Conformation & anti & anti \\
\hline $\begin{array}{c}\tau_{0}\left[\mathrm{C}\left(4^{\prime}\right)-\mathrm{O}\left(1^{\prime}\right)-\right. \\
\left.\mathrm{C}\left(1^{\prime}\right)-\mathrm{C}\left(2^{\prime}\right)\right]\end{array}$ & 18.2 & -3.1 \\
\hline $\begin{array}{r}\tau_{1}\left[\mathrm{O}\left(1^{\prime}\right)-\mathrm{C}\left(1^{\prime}\right)-\right. \\
\left.\mathrm{C}\left(2^{\prime}\right)-\mathrm{C}\left(3^{\prime}\right)\right]\end{array}$ & -34.2 & 22.2 \\
\hline $\begin{array}{l}\tau_{2}\left[C\left(1^{\prime}\right)-C\left(2^{\prime}\right)-\right. \\
\left.C\left(3^{\prime}\right)-C\left(4^{\prime}\right)\right]\end{array}$ & 36.2 & -31.8 \\
\hline $\begin{array}{c}\tau_{3}\left[\mathrm{C}\left(2^{\prime}\right)-\mathrm{C}\left(3^{\prime}\right)-\right. \\
\left.\mathrm{C}\left(4^{\prime}\right)-\mathrm{O}\left(1^{\prime}\right)\right]\end{array}$ & -26.6 & 31.4 \\
\hline $\begin{array}{r}\tau_{4}\left[\mathrm{C}\left(3^{\prime}\right)-\mathrm{C}\left(4^{\prime}\right)-\right. \\
\left.\mathrm{O}\left(1^{\prime}\right)-\mathrm{C}\left(1^{\prime}\right)\right]\end{array}$ & 5.7 & -18.0 \\
\hline Conformation & $\mathrm{C}\left(2^{\prime}\right)-$ exo & $C\left(3^{\prime}\right)-$ exo \\
\hline $\begin{array}{c}\phi_{\mathrm{oo}}\left[\mathrm{O}\left(1^{\prime}\right)-\mathrm{C}\left(4^{\prime}\right)-\right. \\
\left.\mathrm{C}\left(5^{\prime}\right)-\mathrm{O}\left(5^{\prime}\right)\right]\end{array}$ & 175.4 & 161.9 \\
\hline $\begin{array}{c}\phi_{\mathrm{oc}}\left[\mathrm{C}\left(3^{\prime}\right)-\mathrm{C}\left(4^{\prime}\right)-\right. \\
\left.\mathrm{C}\left(5^{\prime}\right)-\mathrm{O}\left(5^{\prime}\right)\right]\end{array}$ & -68.2 & -80.5 \\
\hline Conformation & $\operatorname{tg}^{-}$ & $\operatorname{tg}^{-}$ \\
\hline
\end{tabular}


Fig. 3. Intermolecular hydrogen bondings among molecules A and B, and water.

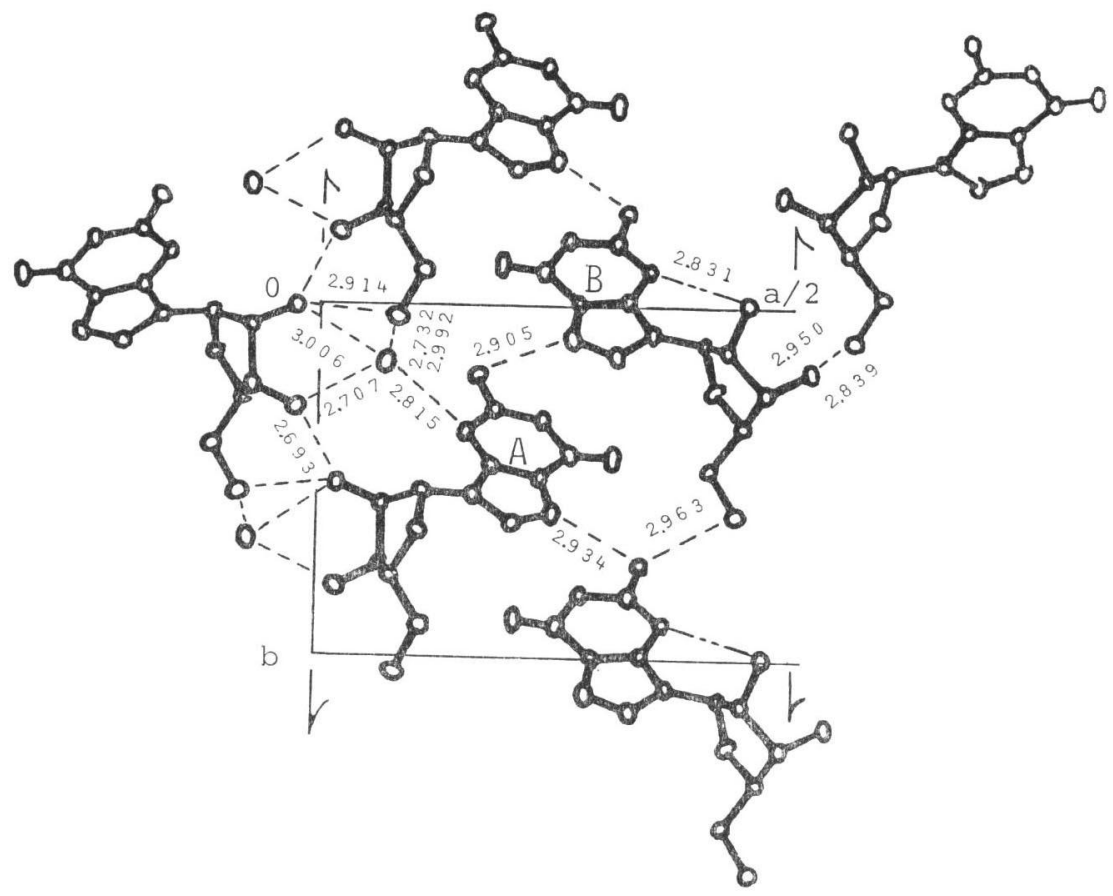

ing water molecules. Thus, the hydrogen bond $\mathrm{O}\left(12^{\prime}\right) \ldots \mathrm{N}(14)$ at $\mathrm{x}, \mathrm{y}, 1+\mathrm{z}(2.831 \AA)$ may cause the $\chi$ angle of the $\mathrm{B}$ molecule to be very large compared with that of $\mathrm{A}$ in which no such hydrogen bond is found (Fig. 3, The numbers of the atoms in the B molecule are those of the corresponding atoms of $\mathrm{A}$ molecule plus 10). It is also noteworthy that the hydrogen bonds involving the $\mathrm{O}\left(2^{\prime}\right) \mathrm{H}$ hydroxyl group are quite different in the A and B molecules. In the latter, $\mathrm{O}\left(12^{\prime}\right)$ is linked to $\mathrm{N}(14)$ in a lateral direction while in the former, $\mathrm{O}\left(2^{\prime}\right)$ links to water, with $\mathrm{O}\left(5^{\prime}\right)$ and $\mathrm{O}\left(3^{\prime}\right)$ forming a rather extended hydrogen bond network. These differences may be responsible for the differences in $\chi$ angles and furanose ring conformations.

\section{Hikaru NAKAMURa NAOMASA Yagisawa NOBUYOSHI SHIMADA TOMOHISA TAKITA \\ Hamao Umezawa *YoICHI IITAKA}

Institute of Microbial Chemistry
Kamiosaki, Shinagawa-ku, Tokyo 141, Japan

*Faculty of Pharmaceutical Sciences, University of Tokyo Hongo, Bunkyo-ku, Tokyo 113 Japan

(Received April 4, 1981)

\section{References}

1) Shimada, N.; N. Yagisawa, H. Naganawa, T. Takita, M. Hamada, T. TaKeuch \& H. UmeZAWA: Oxanosine, a novel nucleoside from actinomycetes. J. Antibiotics 34: 1216 1218, 1981

2) Main, P.; M. M. Woolfson \& G. Germain: "MULTAN, A Computer Program for the Automatic Solution of Crystal Structures," University of York (England) and Leuven (Belgium), 1971

3) Johnson, C.K.: " ORTEP, Oak Ridge National Laboratory Report ORNL-3794," 1965

4) Sundaralingam, M. \& E. Westhof: The "rigid" nucleotide concept in perspective. International J. Quant. Chem., Quantum Biol. Symp. Vol. 6, pp. 115 130, John Wiley \& Sons, Inc., 1979 